\section{Biodiesel und pflanzliche Öle als Kraftstoffe - aus der Nische in den Kraftstoffmarkt}

\author{
von Dieter Bockey, Union zur Förderung \\ von Oel- und Proteinpflanzen e.V. (UFOP)
}

Im Bereich der Rohstoff- und Biokraftstoffproduktion hat sich in Deutschland inzwischen ein komplexer und durch stetiges Wachstum gekennzeichneter Sektor entwickelt. Die Rohstoffnachfrage bei Raps für die Produktion von Biodiesel bestimmt maßgeblich die Anbau- und Preisentwicklung für Ölsaaten in Deutschland und zunehmend in der Europäischen Union. Die Anlagenkapazitäten befinden sich ebenfalls in weiteren Mitgliedstaaten in der Europäischen Union im Aufbau. Es wird erwartet, dass analog wie in Deutschland hiermit einhergehend die Anbaufläche für die Produktion von Ölsaaten zu Lasten der Getreideanbaufläche zunehmen wird. Gleichzeitig eröffnet sich mit der Produktion von Getreide für die Herstellung von Bioethanol ein weiterer zusätzlicher volumenträchtiger Absatzmarkt.

\section{Einleitung}

Als im Dezember 1990 die „Union zur Förderung von Oel- und Proteinpflanzen e.V.“ (UFOP) gegründet wurde, war Biodiesel ein „no-name“-Produkt. Die UFOP mit ihrer interprofessionell ausgerichteten Struktur setzte von Anfang an auf diesen Alternativkraftstoff. Denn angesichts eines soeben verlorenen Soja-Panels im GATT (heute WTO) und der sich abzeichnenden Einführung der Flächenstilllegung zur Reduzierung der Überschüsse bei Getreide und zur Konsolidierung des EU-Haushalts im Rahmen der ersten Stufe der Reform der Gemeinsamen Agrarpolitik (GAP), musste konsequenterweise ein neuer volumenträchtiger Markt entwickelt werden. Es waren die Mitgliedsstaaten Deutschland und Frankreich, die von Anfang an im Rapsanbau auf Stilllegungsflächen zur Produktion von Biodiesel eine neue Absatzalternative sahen. Strategisch trennten sich jedoch schnell die Wege. Frankreich setzte von Beginn an auf die Zumischung von Biodiesel zu Dieselkraftstoff im Rahmen von Pilotvorhaben und hatte bereits im Jahr 1995 eine Produktionskapazität von etwa 250.000 t. Im Gegensatz dazu hat Deutschland von dieser nationalen Ermächtigung gemäß Mineralölsteuerstrukturrichtlinie nicht Gebrauch gemacht, sondern tolerierte die Markteinführung von Biodiesel als Reinkraftstoff, denn eine nationale und von der EUKommission notifizierte Regelung existierte nicht. Erschwerend für die Markteinführung kam hinzu, dass die Verbraucher Biodiesel praktisch nicht kannten oder die Verwendbarkeit in herkömmlichen Dieselmotoren hinterfragt wurde. Die Absatzmengen waren in der Anfangsphase vergleichsweise gering. Erst im September 1995 ging in Leer die erste industrielle Biodieselanlage in Betrieb. Aber immer noch war der Preisunterschied zwischen Biodiesel und Dieselkraftstoff zu gering, um einen Nachfrageschub auszulösen. Erst mit der Freigabenerteilung für alle Diesel-Pkw im Jahr 1995 durch die Volkswagen AG wurde die Voraussetzung geschaffen, ein Kundenpotenzial für die Tankstellen aufzubauen - das „Henne-Ei-Problem“ war motortechnisch gelöst.

\section{Gesetzliche Rahmenbedingungen}

Den ersten Biodieselnachfrage- und nachfolgenden Investitionsboom löste im Jahr 2000 die Einführung der Ökosteuer in drei Stufen mit je $3 €$-Cent pro Liter aus. Biodiesel war der Gewinner der ökologischen Steuerreform, aber steuerrechtlich nicht im Mineralölsteuergesetz verankert. Mit Beschlussfassung der Änderung des Mineralölsteuergesetzes zum 1. Januar 2004 zur Steuerbegünstigung von Biokraftstoffen hat Deutschland eine gesetzlich verbindliche Regelung für Biokraftstoffe geschaffen. Die bisherige Markteinführung und der Kapazitätsaufbau für die Produktion von Biodiesel basierten letztendlich auf einer Protokollerklärung Deutschlands gegenüber der EU-Kommission. Angesichts des Kapazitätszuwachses für die Produktion von Biodiesel bestand Handlungsbedarf zur Legalisierung der Steuerbegünstigung von Biokraftstoffen in Deutschland. Die EURichtlinie zur Förderung von Biokraftstoffen (2003/30/EG) sowie die Energiesteuerrichtlinie (2003/96/EG) gaben zum einen den Handlungsdruck vor, Biokraftstoffe - auf Basis von Mengenzielen - zu einem strategischen Element der Kraftstoffversorgung zu entwickeln und schufen zum anderen den Ermächtigungs- 
spielraum zur Steuerbegünstigung von Biokraftstoffen auf nationaler Ebene.

Die im Biomasseaktionsplan von der EU vorgegebenen Mengenziele, den Biokraftstoffanteil heizwertbereinigt ab dem Jahr 2005 von $2 \%$ auf $5,75 \%$ bis zum Jahr 2010 steigen zu lassen, sind sehr ambitioniert, gemessen an dem hierfür erforderlichen Rohstoffbedarf. Dem Antrag Deutschlands auf Gewährung nationaler Beihilfen - für die Mineralölsteuerbefreiung für Biokraftstoffe - hatte die EUKommission am 18. Februar 2004 zugestimmt und als befristete Maßnahme für den Zeitraum vom 1. Januar 2004 bis zum 31. Dezember 2009 genehmigt.

Deutschland hatte zu diesem Zeitpunkt als Voraussetzung für die Erteilung der vollständigen Steuerbefreiung für Biokraftstoffe vermitteln können, dass im Falle einer vollständigen Mineralölsteuerbefreiung die Produktionskosten für Biokraftstoffe ohne Verbrauchssteuer nicht unter dem Marktpreis einschließlich Verbrauchssteuer von herkömmlichen Kraftstoffen liegen. Die EU-Kommission hatte als Ergebnis der Notifizierung anerkannt, dass nicht nur die Produktionskosten berücksichtigt werden müssen, sondern kostensteigernd auch der entsprechende Mehrverbrauch bei Biokraftstoffen (Reinkraftstoffnutzung) aufgrund des niedrigeren Energiegehaltes. Dieser „Kostenfaktor“ entfällt im Falle der Zumischung zu Dieselkraftstoff (max. $5 \%$ gemäß DIN EN 590) oder Benzin (max. $5 \%$ gemäß DIN EN 228) auf der Stufe der Mineralölindustrie. Bei Biodiesel und Bioethanol kam die Kommission zu dem Ergebnis, dass die nationale Mineralölsteuerbefreiung nicht zu einer Überkompensation führt. Die Kommission erkannte die im nationalen Mineralölsteuergesetz festgelegten Maßnahmen zur jährlichen Prüfung auf Überkompensation im Wege der Berichterstattung an das Parlament an. Die Kommission stellte fest, dass sich die Beihilfe darauf beschränkt, die Differenz zwischen den Produktionskosten der Biokraftstoffe und deren Marktpreis zu kompensieren. Diese Situation hat sich durch die Rohölpreiserhöhung 2004 und besonders 2005 geändert.

Im ersten Bericht der Bundesregierung an den Bundestag zur Überkompensationsprüfung (vgl. Tab. 1) vom 21. Juni 2005 (Deutscher Bundestag 2005) hatte die Bundesregierung anerkannt, dass es darüber hinaus für den
Marktzugang eines Preisanreizes bedarf (s. auch Beispiel Erdgas), und konsequenterweise empfohlen, diese Notwendigkeit bei der Abstimmung für einen Vorschlag einer Teilbesteuerung zu berücksichtigen.

Tab.1: Überkompensationsprüfung bei Biodiesel

\begin{tabular}{|c|c|c|}
\hline & \multicolumn{2}{|c|}{ Biodieseleinsatz als } \\
\hline & $\begin{array}{c}\text { Rein- } \\
\text { kraftstoff } \\
(€-\text { Cent/l) }\end{array}$ & $\begin{array}{c}\text { Beimi- } \\
\text { schung } \\
(€-C e n t / l)\end{array}$ \\
\hline Rapsölpreis frei Ölmühle & 49 & 49 \\
\hline Raffination & 4 & 4 \\
\hline $\begin{array}{l}\text { Veresterung abzgl. } \\
\text { Glyceringutschrift }\end{array}$ & 7 & 7 \\
\hline Beimischungskosten & - & 3 \\
\hline Logistik & 8 & 8 \\
\hline Technischer Mehraufwand & 3 & - \\
\hline Mehrverbrauch & 5 & - \\
\hline Summe (ohne USt) & 76 & 71 \\
\hline $\begin{array}{l}\text { Durchschnittspreis } \\
\text { Diesel } 2004\end{array}$ & 81 & 81 \\
\hline Überkompensation & 5 & 10 \\
\hline
\end{tabular}

Quelle: Deutscher Bundestag 2005

Das Inkrafttreten des Mineralölsteueränderungsgesetzes zum 1.1.2004 hat in Deutschland einen regelrechten Investitionsboom ausgelöst und in der Mineralölindustrie zu einer entsprechenden Nachfrage nach Biokraftstoffen geführt.

\section{Produktions- und Absatzentwicklung}

Die Biodieselproduktionskapazität hat sich bis Ende 2005 auf rund 2,3 Mio. t erhöht und damit gegenüber dem Jahr 2000 mehr als verzehnfacht (vgl. Abb. 1 nächste Seite) und wird sich bis Ende 2006 unter Berücksichtigung der sich zurzeit in der Planung befindlichen Anlagen auf drei bis vier Mio. $t$ erhöhen. In den vergangenen Jahren wurden ca. 400 bis 500 Mio. $€$ in den Aufbau der Biodieselproduktion investiert. Deutschland ist weltweit führend in der Produktion von Biodiesel und hiermit einhergehend in der Anlagentechnologieentwicklung und in der Entwicklung der erforderlichen Fahrzeugkonzepte für den Betrieb mit Biodiesel als Reinkraftstoff. Es müssen nicht nur die materialtechnischen Eigenschaften, sondern insbesondere 
die steigenden emissionsrechtlichen Anforderungen als Voraussetzung für die Freigabenerteilung erfüllt werden. Die DaimlerChrysler AG erteilt inzwischen sogar für die Abgasstufe EURO 5 in Verbindung mit einer Sonderausstattung die Freigabe für Nutzfahrzeuge. ${ }^{1}$

\section{Abb. 1: Biodieselkapazitäten in Deutschland}

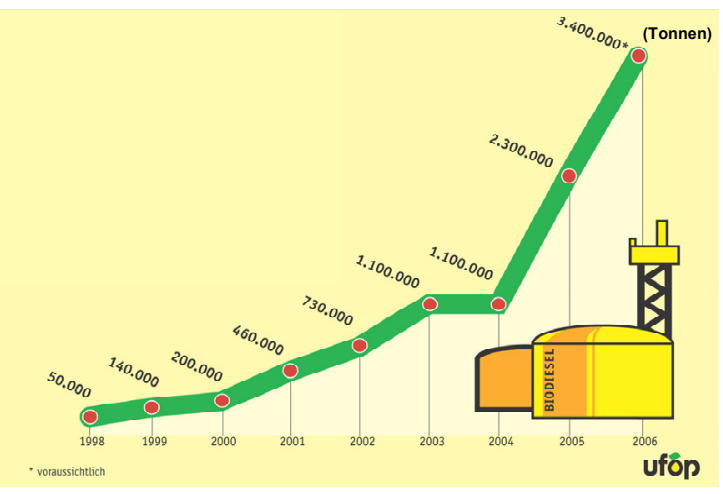

Konsequenterweise wird ebenfalls die Ölmühlenkapazität von zurzeit ca. 5,5 Mio. t bis Ende 2007 auf 7,5 Mio. $t$ ausgebaut. Weitere Ölmühlen, die in Anlagenkonzepte für Biodieselanlagen integriert sind, befinden sich in der Planungsphase. Die nachhaltige Versorgung mit Rapsöl als Rohstoff ist ein entscheidendes strategisches Element zur Wettbewerbssicherung von Biodieselanlagen - insbesondere in Jahren, in denen witterungsbedingt die Ernte unter den Erwartungen bleibt und hohe Pflanzenölpreise bzw. die reduzierte Verfügbarkeit von Rapsöl die Existenz der Anlagen gefährden. Die Insolvenz der Biodieselanlage am Standort Schwarzheide / Brandenburg im Jahr 2003 ist ein Beispiel, das sich nicht wiederholen sollte.

Auch in Kleinpressanlagen zur Gewinnung von Rapsöl wurde erheblich investiert - schätzungsweise 60 bis 70 Mio. $€$. In den vergangenen drei Jahren stieg die Anzahl dezentraler Abpressanlagen geradezu rasant von 98 auf ca. 300 an, die heute insgesamt eine jährliche Vermahlungskapazität von 0,4 bis 0,5 Mio.t Rapssaat erreichen. Hergestellt wird vorrangig Rapsöl zur Kraftstoffnutzung oder als Rohstoff für die Biodieselproduktion. Die UFOP geht davon aus, dass sich die Kooperation zwischen den Biodieselherstellern insbesondere dann erheblich intensivieren wird, wenn auch in Zukunft die
Verwendung von Pflanzenölkraftstoffen in der Land- und Forstwirtschaft dauerhaft von der Mineralölsteuer befreit bleibt. Für die Nutzung von Rapsölkraftstoff existiert eine mit finanzieller Förderung der UFOP entwickelte Vornorm E DIN V 51605, die bis Mitte 2006 in eine endgültige nationale Norm überführt werden soll.

Einhergehend mit der Kapazitätsentwicklung erreichte der Biodieselabsatz im Jahr 2005 mit insgesamt 1,8 Mio. $t$ erneut einen Höchstwert (vgl. Abb. 2). Es wird geschätzt, dass davon rund 1,5 Mio. $t$ aus inländischer Produktion stammen. Verlässliche statistische Angaben liegen hierzu nicht vor. Ursache ist die (noch) fehlende Einbindung der Biokraftstoffe in die entsprechende Mineralölstatistik.

\section{Abb. 2: Entwicklung des Biodieselabsatzes in Deutschland}

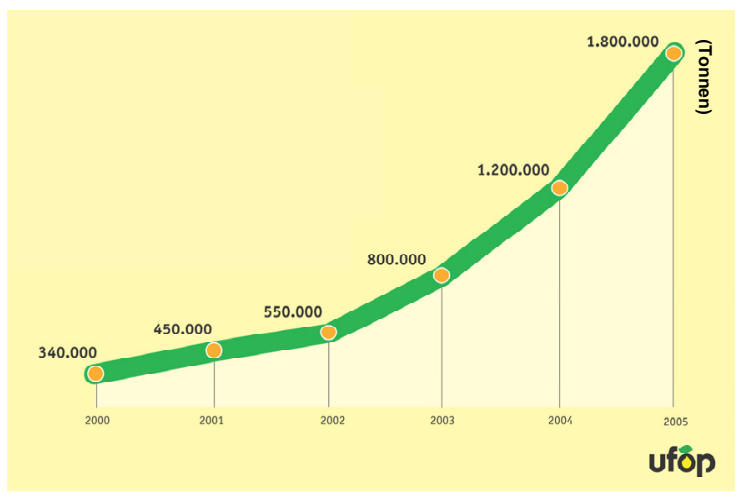

Heute bieten mehr als 500 Unternehmen des Tankstellenmittelstandes (konzernunabhängig) an etwa 1.900 öffentlichen Tankstellen - jede Neunte im Bundesgebiet - praktisch flächendeckend Biodiesel an. Nach einer aktuellen Erhebung der Arbeitsgemeinschaft Qualitätsmanagement Biodiesel e.V. (2006) haben die Tankstellenbetreiber allein 2005 etwa 8 Mio. $€$ und seit 1996 insgesamt ca. 35 Mio. $€$ in die Errichtung und Umrüstung öffentlicher Tankstellen investiert. Für diese Unternehmen hat sich Biodiesel als wichtige zusätzliche Einkommensquelle in einem hart umkämpften Kraftstoffmarkt entwickelt. Die Absatzmenge lag im Jahr 2005 bei etwa $520.000 \mathrm{t}$ (in 2004 bei ca. $420.000 \mathrm{t}$ ) (vgl. Abb. 3 nächste Seite). 


\section{Abb. 3: Absatz und Verwendung von Biodiesel in Deutschland im Jahr 2005}

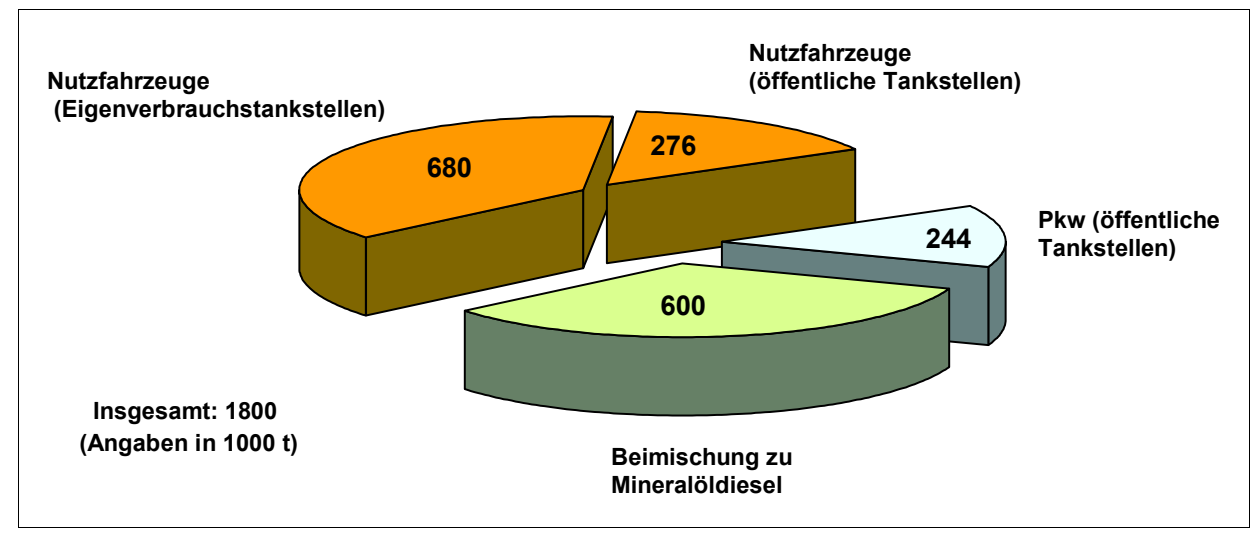

Quelle: AGQM 2006

Etwa 1 Mio. t Biodiesel wurden 2005 als Reinkraftstoff (einschließlich Verkauf über öffentliche Tankstellen) direkt an das Speditionsgewerbe, den öffentlichen Personennahverkehr und an die Landwirtschaft verkauft. Das Absatzpotenzial in der Land- und Forstwirtschaft wird unter Berücksichtigung der gegebenen Regelung für die Agrardieselrückvergütung (10.000 Litergrenze) auf etwa 300.000 bis $400.000 \mathrm{t}$ geschätzt. Etwa $600.000 \mathrm{t}$ Biodiesel wurden auf der Stufe der Mineralölindustrie dem Dieselkraftstoff zugemischt. Eine Beimischung von bis zu $5 \%$ sind gemäß DIN EN 590 erlaubt.

\section{Auswirkungen auf die Landwirtschaft in Deutschland}

Durch die Rohstoffproduktion für Biodiesel wurden im Jahr 2005 bereits 1 Mio. ha Anbaufläche in Deutschland gebunden (vgl. Tab. 2). Das gesamte Anbauflächenpotenzial wird auf 1,6 bis maximal 1,8 Mio. ha geschätzt. Davon werden rund 400.000 ha für die Produktion von Rapsöl für die Nahrungsmittelindustrie und etwa 100.000 ha für die Bereiche Oleochemie ${ }^{2}$ und Schmierstoffe benötigt.

Tab. 2: Anbaustatistik für nachwachsende Rohstoffe in Deutschland

\begin{tabular}{|c|c|c|c|c|c|c|c|c|c|}
\hline \multirow[b]{2}{*}{ Art der Fläche } & \multicolumn{2}{|c|}{2003} & \multicolumn{3}{|c|}{2004} & \multicolumn{3}{|c|}{2005} & \multirow{2}{*}{$\begin{array}{c}2005 \\
\begin{array}{c}\text { Energiepflan- } \\
\text { zen }\end{array}\end{array}$} \\
\hline & Basis & $\begin{array}{c}\text { Still- } \\
\text { legung }\end{array}$ & Basis & $\begin{array}{c}\text { Still- } \\
\text { legung }\end{array}$ & $\begin{array}{l}\text { Energie- } \\
\text { pflanzen }\end{array}$ & Basis & $\begin{array}{c}\text { Still- } \\
\text { legung }\end{array}$ & $\begin{array}{l}\text { Energie- } \\
\text { pflanzen }\end{array}$ & \\
\hline & \multicolumn{8}{|c|}{ Deutschland $^{l)}($ ha) } & $\begin{array}{c}\text { EU-Importe } \\
\text { (ha) }\end{array}$ \\
\hline Stärke & 125.000 & 0 & 125.000 & 0 & 0 & 125.000 & 0 & - & \\
\hline Zucker & 7.000 & 0 & 7.000 & 0 & 0 & 7.000 & 0 & - & \\
\hline Rapsöl & 340.000 & 328.753 & 424.000 & 210.000 & 80.800 & 561.665 & 322.000 & 121.926 & 130.669 \\
\hline Sonnenblumenöl & 15.000 & 3.983 & - & 750 & 0 & - & 1.900 & - & \\
\hline Getreide $^{2)}$ & - & - & - & 3.400 & 31.500 & - & 43.534 & 118.531 & \\
\hline Leinöl & 5.000 & 365 & 3.000 & 100 & 0 & 0 & 0 & - & \\
\hline Faserpflanzen & 2.800 & 0 & 2.000 & 0 & 0 & 1.500 & 75 & - & \\
\hline Heilstoffe & 4.000 & 693 & 4.000 & 400 & 0 & 4.000 & 182 & - & \\
\hline Sonstiges & - & 5.051 & - & 350 & 0 & - & 2.811 & 2.320 & \\
\hline Summe & 498.800 & 338.045 & 565.000 & 215.000 & 112.300 & 699.165 & 370.502 & 242.777 & 130.669 \\
\hline insgesamt & \multicolumn{2}{|c|}{836.845} & \multicolumn{3}{|c|}{893.300} & \multicolumn{3}{|c|}{1.312 .444} & \\
\hline
\end{tabular}


Die Anbaufläche von Raps hat sich mit 1,4 Mio. ha (geschätzt für die Ernte 2006) innerhalb von 15 Jahren praktisch verdoppelt. Diese Anbauentwicklung hat erheblich dazu beigetragen, die Überschusssituation im Getreidebereich und damit die Kosten für staatliche Interventionen zur Marktregulierung zu reduzieren.

Insbesondere in den Ackerbaubetrieben der neuen Bundesländer hat sich der Rapsanbau als eine alternative Blattfrucht zur Zuckerrübe in Getreidefruchtfolgen entwickelt. Bei Rapserzeugerpreisen zur Ernte 2005 zwischen 200 und $210 € / \mathrm{t}$ und einer Erntemenge von ca. 3,8 Mio. $t$ wird mit diesem Produktionszweig inzwischen ein Bruttoumsatz in der Landwirtschaft von etwa 0,76 Mrd. $€$ erzielt.

Mit dem geschätzten Anbauflächenpotenzial von etwa 1,4 Mio. ha Raps für die Biodieselproduktion verfügt Deutschland über das erforderliche Rohstoffpotenzial zur Erreichung des Mengenziels gemäß EU-Aktionsplan im Jahr 2010, nach dem mindestens 5,75\% des Dieselmarktes mit Biodiesel (ca. 2 Mio. t) abgedeckt werden sollen (vgl. Tab. 3). Zur Sicherung der Rohstoffbeschaffung aus der EU bzw. Drittländern orientiert sich die aktuelle Standortplanung für Neuanlagen entlang schiffbarer Binnengewässer bzw. Häfen (z. B. Regensburg, Höchst, Mainz, Brunsbüttel, Rostock, Halle, Neuss, Magdeburg).

Tab. 3: Zielvorgaben der EU-Biokraftstoffrichtlinie, bezogen auf Deutschland

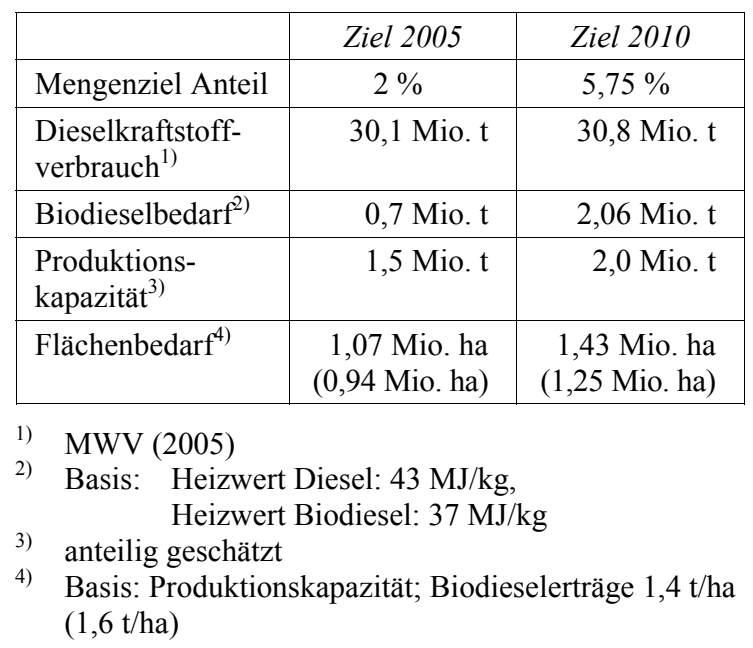

\section{Flächen- und Mengenpotenziale in der EU}

Das Rohstoffpotenzial für die Biodieselproduktion ist jedoch begrenzt. In der EU-25 wurden 2004 auf ca. 7 Mio. ha rund 19,2 Mio. t Ölsaaten (Raps: 15,1 Mio.t, Sonnenblumen: 4,1 Mio.t) geerntet; dies entspricht einer Ölmenge von etwa 8 Mio.t. Gemessen am Dieselbedarf der EU-25 im Jahr 2010 (165 Mio. t) würden heizwertbereinigt etwa 11 Mio. t Biodiesel benötigt (vgl. Tab. 4). Der Vergleich macht deutlich, dass das Mengenziel von 5,75\% für das Jahr 2010 ausschließlich auf Basis europäischer Rohstoffe nicht erreicht werden kann. Die Einführung eines EU-weiten Beimischungszwangs (s. Österreich, Niederlande, Großbritannien, Deutschland) würde konsequenterweise zu einem Anstieg der Importe pflanzlicher Öle und von Biodiesel aus Drittländern führen.

Da die EU-Richtlinie zur Förderung von Biokraftstoffen keine Mengenvorgaben für die jeweiligen Kraftstoffarten macht, kann diese „Lücke“ durch die Produktion von Bioethanol kompensiert werden. Im Vergleich zur Produktion von Biodiesel aus Ölsaaten ist das Rohstoffpotenzial für die Herstellung von Bioethanol in der EU weitaus größer (siehe Beitrag von Schmitz in diesem Schwerpunkt).

Tab. 4: Zielvorgaben der EU-Biokraftstoffrichtlinie, bezogen auf die EU-25

\begin{tabular}{|c|c|c|c|}
\hline & & $\begin{array}{l}\text { Ziel } \\
2005\end{array}$ & $\begin{array}{l}\text { Ziel } \\
2010\end{array}$ \\
\hline Mengenziel Anteil & & $2 \%$ & $5,75 \%$ \\
\hline $\begin{array}{l}\text { Dieselkraftstoff- } \\
\text { verbrauch }^{1)}\end{array}$ & (Mio. t) & 158,6 & $165,0^{4)}$ \\
\hline Biodieselbedarf $^{2)}$ & (Mio. t) & 3,69 & 11,0 \\
\hline Flächenbedarf ${ }^{3)}$ & (Mio. ha) & 2,63 & 7,88 \\
\hline $\begin{array}{l}\text { Ottokraftstoff- } \\
\text { verbrauch }{ }^{1)}\end{array}$ & (Mio. t) & 124,8 & $113,6^{4)}$ \\
\hline Ethanolbedarf ${ }^{2)}$ & (Mio. t) & 3,7 & 9,7 \\
\hline Flächenbedarf ${ }^{3)}$ & (Mio. ha) & 1,85 & 4,84 \\
\hline Gesamtfläche & (Mio. ha) & 4,48 & 12,72 \\
\hline \multicolumn{4}{|c|}{ EUROSTAT (2002) } \\
\hline \multicolumn{4}{|c|}{$\begin{array}{l}\text { 2) Basis: Heizwert Diesel: } 43 \mathrm{MJ} / \mathrm{kg} \text {, Heizwert Biodie- } \\
\text { sel: } 37 \mathrm{MJ} / \mathrm{kg} \text {, Heizwert Normalbenzin: } 40 \mathrm{MJ} / \mathrm{kg} \text {, } \\
\text { Heizwert Ethanol: } 27 \mathrm{MJ} / \mathrm{kg} \\
\text { 3) } \\
\text { 4iodieselerträge } 1,4 \mathrm{t} / \mathrm{ha} \text {, Bioethanolertrag } 2 \mathrm{t} / \mathrm{ha} \\
\text { Annahmen: Rückgang Verbrauch Ottokraftstoffe } 9 \% \text {, } \\
\text { Zuwachs Verbrauch Dieselkraftstoff } 4 \%\end{array}$} \\
\hline
\end{tabular}


Unter Berücksichtigung des Stands der Konversionstechnik und der vorhandenen Biomassepotenziale ergibt sich ein theoretisches Bioethanolpotenzial in der EU-25 von ca. 20 Mio. $t$ und in Deutschland von ca. 6,3 Mio. t. ${ }^{3}$

Letztere Angabe entspricht - bei Zugrundelegung der aktuellen Verbrauchsprognose des Mineralölwirtschaftsverbandes für Ottokraftstoff von 22 Mio. $t$ im Jahr 2010 - einem Substitutionspotenzial von rund $29 \%$.

\section{Zusammenfassung}

Es kann festgestellt werden, dass sich im Bereich der Rohstoff- und Biokraftstoffproduktion inzwischen ein komplexer und durch stetiges Wachstum gekennzeichneter Sektor in Deutschland entwickelt hat. Die Rohstoffnachfrage bei Raps für die Produktion von Biodiesel bestimmt maßgeblich die Anbau- und Preisentwicklung für Ölsaaten in Deutschland und zunehmend in der Europäischen Union. Anlagenkapazitäten befinden sich ebenfalls in weiteren Mitgliedstaaten in der Europäischen Union im Aufbau. Es wird erwartet, dass analog wie in Deutschland hiermit einhergehend die Anbaufläche für die Produktion von Ölsaaten zu Lasten der Getreideanbaufläche zunehmen wird.

Gleichzeitig eröffnet sich mit der Produktion von Getreide für die Herstellung von Bioethanol ein weiterer zusätzlicher volumenträchtiger Absatzmarkt. In Deutschland und in der Europäischen Union besteht ein erhebliches Rohstoffpotenzial, so dass Bioethanol auch als eine mengenwirksam bedeutende Kraftstoffalternative nach dem Vorbild Brasiliens entwickelt werden könnte. Die Kraftstoff- und Forschungsstrategie der letzten Bundesregierung setzt jedoch bei der marktfernen und auch in den strukturellen Auswirkungen bisher nicht ausreichend bewerteten Produktion von synthetischen Kraftstoffen aus Biomasse an. Hier besteht Handlungsbedarf zur Anpassung der Biokraftstoffstrategie als Element der nationalen Nachhaltigkeitsstrategie.

\section{Anmerkungen}

1) Siehe dazu die Angaben auf der Homepage von UFOP (http://www.ufop.de).

2) Unter der Oleochemie versteht man die Chemie von Fetten und Ölen.
3) Persönliche Mitteilung gegenüber dem Autor durch die „Landwirtschaftliche Arbeitsgruppe Biokraftstoffe" (2006)

\section{Literatur}

AGQM - Arbeitsgemeinschaft Qualitätsmanagement Biodiesel e.V., 2006: Steigender Absatz von Biodiesel für Nutzfahrzeuge; http://www.agqmbiodiesel.de/_1_51.html (letzter Zugang: 27.03.2006)

BLE - Bundesanstalt für Landwirtschaft und Ernährung, 2005: Anbaustatistik für nachwachsende Rohstoffe; http://www.ble.de/

Deutscher Bundestag, 2005: Bericht zur Steuerbegünstigung für Biokraft- und Bioheizstoffe. BTDrucksache 15/5816 vom 21.06.2005

EUROSTAT, 2002: http://epp.eurostat.cec.eu.int MWV - Mineralölwirtschaftsverband, 2005: MWVPrognose 2020 für die Bundesrepublik Deutschland; http://www.mwv.de/Download/prognose.pdf (letzter Zugang: 27.03.2006)

\section{Kontakt}

Dieter Bockey

Union zur Förderung von Oelund Proteinpflanzen e.V. (UFOP)

Claire-Waldoff-Straße 7, 10117 Berlin

Tel.: +49 (0) 30 / 31904 - 215

E-Mail: d.bockey@ufop.de 